\title{
High Energy Neutrinos from Gamma-Ray Bursts
}

\author{
Eli Waxman * † a \\ ${ }^{a}$ Dept. of Condensed Matter Physics, Weizmann Institute of Science, Rehovot 76100, Israel
}

\begin{abstract}
Observations suggest that $\gamma$-ray bursts (GRBs) are produced by the dissipation of the kinetic energy of a relativistic fireball. In this talk, recent work on the production of high energy neutrinos by GRB fireballs is reviewed. A significant fraction of GRB energy is expected to be converted to an accompanying burst of high energy neutrinos. Photomeson interactions produce a burst of $\sim 100 \mathrm{TeV}$ neutrinos in coincidence with the GRB, and a burst of $\sim 10^{18} \mathrm{eV}$ neutrinos following the GRB on a time scale of $10 \mathrm{~s}$. Inelastic $p-n$ nuclear collisions result in the production of a burst of $\sim 10 \mathrm{GeV}$ neutrinos in coincidence with the GRB. Planned $1 \mathrm{~km}^{3}$ neutrino telescopes are expected to detect tens of $100 \mathrm{TeV}$ neutrino events, and several $10^{18} \mathrm{eV}$ events, correlated with GRBs per year. A suitably densely spaced detector may allow the detection of several $10 \mathrm{GeV}$ events per year. The detection of high-energy neutrino events correlated with GRBs will allow to constrain GRB progenitor models and to test the suggestion that GRBs accelerate protons to $\sim 10^{20} \mathrm{eV}$. Moreover, such detection will allow to test for neutrino properties, e.g. flavor oscillations (for which upward moving $\tau$ 's would be a unique signature) and coupling to gravity, with an accuracy many orders of magnitude better than is currently possible.
\end{abstract}

\section{Introduction}

The widely accepted interpretation of the phenomenology of GRBs, bursts of $0.1 \mathrm{MeV}-1 \mathrm{MeV}$ photons lasting for a few seconds [1], is that the observable effects are due to the dissipation of the kinetic energy of a relativistically expanding wind, a "fireball," whose primal cause is not yet known (see 2 for review). During the past three years, delayed low energy emission (afterglow) of GRBs has been discovered in X-ray, optical, and radio wave bands (see [3] for review). Afterglow observations confirmed the cosmological origin of the bursts, through the redshift determination of several GRB host-galaxies, and confirmed standard model predictions of afterglows that result from the collision of an expanding fireball with its

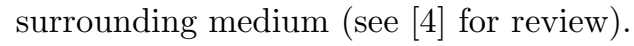

The physical conditions in the fireball dissipation region allow Fermi shock acceleration of protons to energy $>10^{20} \mathrm{eV}$ [5, 6]. The average rate at which energy is emitted as $\gamma$-rays by GRBs is comparable to the energy generation rate of $>10^{19} \mathrm{eV}$ cosmic-rays in a model where such

\footnotetext{
*Incumbent of the Beracha foundation career development chair

†Work supported in part by BSF Grant 9800343, AEC Grant 38/99 and MINERVA Grant.
}

ultra-high energy cosmic-rays (UHECRs) are produced by a cosmological distribution of sources [5,7]. These two facts suggest that GRBs and UHECRs have a common origin (see [8] for review).

Emission of high energy neutrinos is expected to accompany the observed GRB photons, due to photomeson interactions of high energy protons with fireball photons [9, 10, and due to protonneutron inelastic collisions [11 13]. In this review, recent work on the production of high energy neutrinos by GRB fireballs is summarized, and the implications for planned $\sim 1 \mathrm{~km}^{3}$ neutrino telescopes (the ICECUBE extension of AMANDA, ANTARES, NESTOR; see [14] for review) are discussed. The fireball model is briefly described in §2. Proton acceleration in GRB fireballs is discussed in $\S 3$. High energy neutrino production in fireballs and its implications for future high energy neutrino detectors are discussed in $\S 4$.

\section{The fireball model}

In the fireball model of GRBs [15], a compact source, of linear scale $r_{0} \sim 10^{7} \mathrm{~cm}$, produces a wind characterized by an average luminosity $L \sim 10^{52} \mathrm{erg} \mathrm{s}^{-1}$ and mass loss rate $\dot{M}=L / \eta c^{2}$. At small radius, the wind bulk Lorentz factor, $\Gamma$, 
grows linearly with radius, until most of the wind energy is converted to kinetic energy and $\Gamma$ saturates at $\Gamma \sim \eta \sim 300$. Variability of the source on time scale $\Delta t$, resulting in fluctuations in the wind bulk Lorentz factor $\Gamma$ on similar time scale, then leads to internal shocks [16] in the expanding fireball at a radius $r_{i} \approx \Gamma^{2} c \Delta t$. If the Lorentz factor variability within the wind is significant, internal shocks reconvert a substantial part of the kinetic energy to internal energy. It is assumed that this energy is then radiated as $\gamma$-rays by synchrotron and inverse-Compton emission of shockaccelerated electrons.

In this model, the observed $\gamma$-ray variability time, $\sim r_{i} / \Gamma^{2} c \approx \Delta t$, reflects the variability time of the underlying source, and the GRB duration, $T \sim 10$ s, reflects the duration over which energy is emitted from the source. A large fraction of bursts detected by BATSE show variability on the shortest resolved time scale, $\sim 10 \mathrm{~ms}$ [17, and some show variability on shorter time scales, $\sim 1 \mathrm{~ms}$ 18. This sets the constraint on underlying source size, $r_{0}<c \Delta t \sim 10^{7} \mathrm{~cm}$. The wind must be expanding relativistically, with a Lorentz factor $\Gamma \sim 300$, in order that the fireball pairproduction optical depth be small for observed high energy, $100 \mathrm{MeV}$, GRB photons [19].

As the fireball expands, it drives a relativistic shock (blast-wave) into the surrounding gas. At early time, $t \ll T$, the fireball is little affected by this external interaction. At late time, $t \gg T$, most of the fireball energy is transferred to the surrounding gas, and the flow approaches the Blandford-McKee self-similar flow [20]. The shock driven into the ambient medium continuously heats fresh gas, and accelerates relativistic electrons which produce through synchrotron emission the delayed radiation, "afterglow," observed on time scales of days to months. As the shock-wave decelerates, due to accumulation of ambient gas mass, the emission shifts with time to lower frequencies. For expansion into uniform density gas, the shock Lorentz factor decreases with time according to $\Gamma_{B M}(t) \propto t^{-3 / 8}$.

During the transition to self-similar expansion, which takes place on (observed) time scale comparable to $T$, reverse shocks propagate into the fireball ejecta and decelerate it. At this stage the shocked plasma expands with the self-similar Lorentz factor, which for expansion into uniform density gas is given by (e.g. 10])

$\Gamma_{B M}(t=T) \simeq 245\left(\frac{E_{53}}{n_{0}}\right)^{1 / 8} T_{1}^{-3 / 8}$,

while the unshocked fireball ejecta propagate with the original expansion Lorentz factor, $\Gamma=\eta>$ $\Gamma_{B M}(t=T)$. Here, $E=10^{53} E_{53}$ erg is the total fireball energy, $T=10 T_{1} \mathrm{~s}$, and $n=1 n_{0} \mathrm{~cm}^{-3}$ is the ambient gas density. $n_{0}=1$ is typical to the interstellar medium.

Internal shocks are expected to be "mildly" relativistic in the fireball comoving frame, i.e. characterized by Lorentz factor $\Gamma_{i}-1 \sim 1$ in the wind rest frame, since adjacent shells within the wind are expected to expand with Lorentz factors which do not differ by more than an order of magnitude. Moreover, the reverse shocks are also expected to be mildly relativistic, since the ratio $\eta / \Gamma_{B M}(t=T)$ is not far from unity.

\section{UHECRs from GRB fireballs}

\subsection{Fermi acceleration in GRBs}

In the fireball model, the observed GRB and afterglow radiation is produced by synchrotron emission of shock accelerated electrons. In the region where electrons are accelerated, protons are also expected to be shock accelerated. This is similar to what is thought to occur in supernovae remnant shocks [21]. We consider below proton acceleration in internal and reverse fireball shocks. Since these shocks are mildly relativistic, we expect results related to particle acceleration in sub-relativistic shocks (see, e.g., 21] for review) to be valid for the present scenario. In particular, the predicted energy distribution of accelerated protons is $d N_{p} / d \epsilon_{p} \propto \epsilon_{p}^{-2}$.

Two constraints must be satisfied by fireball wind parameters in order to allow proton acceleration to $\epsilon_{p}>10^{20} \mathrm{eV}$ in internal shocks [5]:

$\xi_{B} / \xi_{e}>0.02 \Gamma_{2.5}^{2} \epsilon_{p, 20}^{2} L_{\gamma, 52}^{-1}$,

and

$\Gamma>130 \epsilon_{p, 20}^{3 / 4} \Delta t_{-2}^{-1 / 4}$. 
Here, $\epsilon_{p}=10^{20} \epsilon_{p, 20} \mathrm{eV}, \Delta t=10^{-2} \Delta t_{-2} \mathrm{~s}, \Gamma=$ $10^{2.5} \Gamma_{2.5}$ is the plasma expansion Lorentz factor, $L_{\gamma}=10^{52} L_{\gamma, 52} \mathrm{erg} / \mathrm{s}$ is the $\gamma$-ray luminosity, $\xi_{B}$ is the fraction of the wind energy density which is carried by magnetic field, $4 \pi r^{2} c \Gamma^{2}\left(B^{2} / 8 \pi\right)=$ $\xi_{B} L$, and $\xi_{e}$ is the fraction of wind energy carried by shock accelerated electrons. To obtain the constraints for acceleration in reverse shocks, $\Delta t$ should be replaced by $T$. Since the electron energy is lost radiatively, $L_{\gamma} \approx \xi_{e} L$. The first constraint must be satisfied in order for the proton acceleration time $t_{a}$ to be smaller than the wind expansion time. The second constraint must be satisfied in order for the synchrotron energy loss time of the proton to be larger than $t_{a}$.

The constraints that must be satisfied to allow acceleration of protons to energy $>10^{20} \mathrm{eV}$ are remarkably similar to those inferred from $\gamma$-ray observations. $\Gamma>100$ is implied by observed $\gamma$ ray spectra, and magnetic field close to equipartition, $\xi_{B} \sim 1$, is required in order for electron synchrotron emission to account for the observed radiation.

We have assumed in the discussion so far that the fireball is spherically symmetric. However, since a jet-like fireball behaves as if it were a conical section of a spherical fireball as long as the jet opening angle is larger than $\Gamma^{-1}$, our results apply also for a jet-like fireball (we are interested only in processes that occur when the wind is ultra-relativistic, $\Gamma \sim 300$, prior to significant fireball deceleration). For a jet-like wind, $L$ in our equations should be understood as the luminosity the fireball would have carried had it been spherically symmetric.

\subsection{Energy generation rate}

The observed GRB redshift distribution implies a typical GRB $\gamma$-ray energy of $E \approx 10^{53} \mathrm{erg}$, and a GRB rate of $R_{\mathrm{GRB}} \sim 3 / \mathrm{Gpc}^{3} \mathrm{yr}$ at $z \sim 1$. The present, $z=0$, rate is less well constrained, since most observed GRBs originate at redshifts $1 \leq z \leq 2.5$ [3]. Present data are consistent with both no evolution of GRB rate with redshift, and with strong evolution (following, e.g., star formation rate), in which $R_{\mathrm{GRB}}(z=1) / R_{\mathrm{GRB}}(z=0) \sim$ 10 24. The energy observed in $\gamma$-rays reflect the fireball energy in accelerated electrons. If accel-

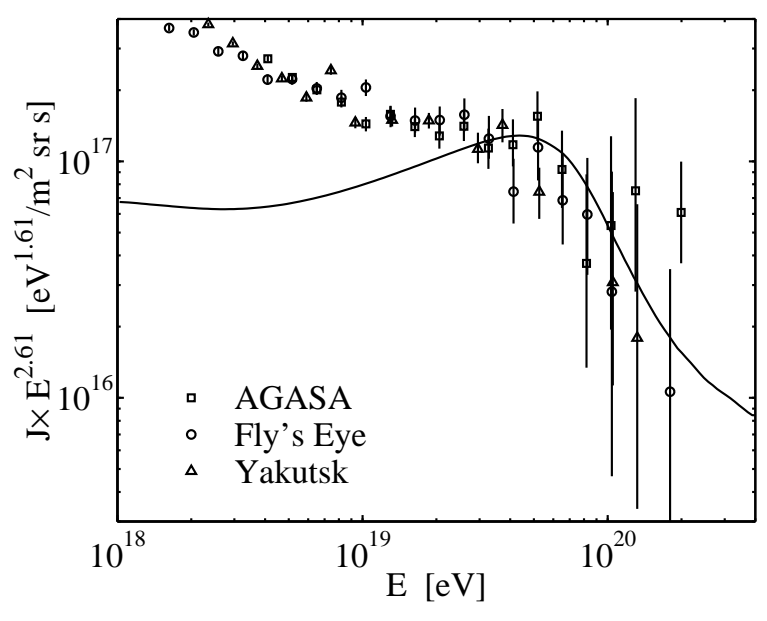

Figure 1. The UHECR flux expected in a cosmological model, where high-energy protons are produced at a rate $\left(\epsilon_{p}^{2} d \dot{n}_{p} / d \epsilon_{p}\right)_{z=0}=0.8 \times$ $10^{44} \mathrm{erg} / \mathrm{Mpc}^{3} \mathrm{yr}$ as predicted in the GRB model [Eq. (4)], compared to the Fly's Eye, Yakutsk and AGASA data. $1 \sigma$ flux error bars are shown. The highest energy points are derived assuming the detected events ( 1 for Fly's Eye and Yakutsk, 4 for AGASA) represent a uniform flux over the energy range $10^{20} \mathrm{eV}-3 \times 10^{20} \mathrm{eV}$.

erated electrons and protons carry similar energy (as indicated by afterglow observations 25]) then the GRB cosmic-ray production rate is

$\epsilon_{p}^{2}\left(d \dot{n}_{p} / d \epsilon_{p}\right)_{z=0}=10^{44} \zeta \mathrm{erg} / \mathrm{Mpc}^{3} \mathrm{yr}$,

with $\zeta$ in the range of $\sim 10^{-0.5}$ to $\sim 10^{0.5}$. This result is consistent with more detailed analyses [26], where $R_{\mathrm{GRB}}(z=0) \times E$ in the range of 0.2 to 2 times $10^{44} \mathrm{erg} / \mathrm{Mpc}^{3} \mathrm{yr}$ is obtained (under the assumption that $R_{\mathrm{GRB}}$ is proportional to star formation rate) for different models of star formation rate evolution.

In Fig. 1 we compare the UHECR spectrum, reported by the Fly's Eye [27], the Yakutsk [28], and the AGASA 29] experiments, with that predicted by the GRB model (assuming GRB rate follows star formation rate). The generation rate (4) of high energy protons is remarkably simi- 
lar to that required to account for the flux of $>10^{19} \mathrm{eV}$ cosmic-rays (The flux at lower energies is most likely dominated by heavy nuclei of Galactic origin [27], as indicated by the flattening of the spectrum at $\approx 10^{19} \mathrm{eV}$ and by the evidence for a change in composition at this energy [30]).

The suppression of model flux above $10^{19.7} \mathrm{eV}$ is due to energy loss of high energy protons in interaction with the microwave background, i.e. to the "GZK cutoff" [31. The available data do not allow to determine the existence (or absence) of the "cutoff" with high confidence. The AGASA results show an excess (at a $\sim 2.5 \sigma$ confidence level) of events compared to model predictions above $10^{20} \mathrm{eV}$. This excess is not confirmed, however, by the other experiments. Moreover, since the $10^{20} \mathrm{eV}$ flux is dominated by sources at distances $<100 \mathrm{Mpc}$, over which the distribution of known astrophysical systems (e.g. galaxies, clusters of galaxies) is inhomogeneous, significant deviations from model predictions presented in Fig. 1 for a uniform source distribution are expected at this energy [7]. Clustering of cosmic-ray sources leads to a standard deviation, $\sigma$, in the expected number, $N$, of events above $10^{20} \mathrm{eV}$, given by $\sigma / N=0.9\left(d_{0} / 10 \mathrm{Mpc}\right)^{0.9}$ [32], where $d_{0}$ is the unknown scale length of the source correlation function and $d_{0} \sim 10 \mathrm{Mpc}$ for field galaxies.

Although the rate of GRBs out to a distance of $100 \mathrm{Mpc}$ from Earth, the maximum distance traveled by $>10^{20} \mathrm{eV}$ protons, is in the range of $10^{-2}$ to $10^{-3} \mathrm{yr}^{-1}$, the number of different GRBs contributing to the flux of $>10^{20} \mathrm{eV}$ protons at any given time may be large. This is due to the dispersion $\tau$ in proton arrival time, which is expected due to deflection by inter-galactic magnetic fields and may be as large as $10^{5} \mathrm{yr}$, implying that the number of sources contributing to the flux at any given time may be as large as $\tau \times 10^{-2} \mathrm{yr}^{-1}=10^{3}$ [7].

\section{High energy Neutrinos}

\subsection{Photomeson production}

4.1.1. Internal shocks neutrinos, $\sim 10^{14} \mathrm{eV}$

The decay of charged pions, $\pi^{+} \rightarrow \mu^{+}+\nu_{\mu} \rightarrow$ $e^{+}+\nu_{e}+\bar{\nu}_{\mu}+\nu_{\mu}$, created in interactions between fireball photons and accelerated protons, results in the production of high energy neutrinos [9]. The key relation is between the observed photon energy, $\epsilon_{\gamma}$, and the accelerated proton's energy, $\epsilon_{p}$, at the photomeson threshold of the $\Delta$-resonance. In the observer frame,

$\epsilon_{\gamma} \epsilon_{p}=0.2 \mathrm{GeV}^{2} \Gamma^{2}$.

For $\Gamma \approx 300$ and $\epsilon_{\gamma}=1 \mathrm{MeV}$, the typical observed energy of photons emitted in internal shocks, we see that characteristic proton energies $\sim 10^{16} \mathrm{eV}$ are required to produce neutrinos from pion decay. Typically, the neutrinos receive $\sim 5 \%$ of the proton energy, leading to neutrinos of $\sim 10^{14} \mathrm{eV}$.

The fraction $f_{\pi}\left(\epsilon_{p}\right)$ of proton energy lost to pion production is given by the ratio of the wind expansion time, $\Gamma \Delta t$ in the wind frame at the internal shock stage, and the proton photopion energy loss time. For observed GRB photon spectra, $d N_{\gamma} / d \epsilon_{\gamma} \propto \epsilon_{\gamma}^{-\beta}$ with $\beta \simeq 1$ for photon energies below the break energy $\epsilon_{\gamma}^{b} \approx 1 \mathrm{MeV}$ and $\beta \simeq 2$ for $\epsilon_{\gamma}>\epsilon_{\gamma}^{b}, f_{\pi}$ is independent of energy for $\epsilon_{p}>0.2 \Gamma^{2} \mathrm{GeV}^{2} / \epsilon_{\gamma}^{b} \sim 10^{16} \mathrm{eV}$

$f_{\pi} \approx 0.2 \frac{L_{\gamma, 52}}{\left(\epsilon_{\gamma}^{b} / 1 \mathrm{MeV}\right) \Gamma_{2.5}^{4} \Delta t_{-2}}$,

and $f_{\pi} \propto \epsilon_{p}$ for $\epsilon_{p}<10^{16} \mathrm{eV}$. For a typical burst, $E \approx 10^{53}$ erg at redshift $z \sim 1$, Eq. (6) implies a neutrino fluence of 9

$\epsilon_{\nu}^{2} \Phi_{\nu_{x}} \approx 10^{-3}\left(\frac{f_{\pi}}{0.2}\right)\left(\frac{\epsilon_{\nu}}{10^{14} \mathrm{eV}}\right)^{\alpha} \frac{\mathrm{GeV}}{\mathrm{cm}^{2}}$,

where $\alpha=0$ for $\epsilon_{\nu}>10^{14} \mathrm{eV}$ and $\alpha=1$ for $\epsilon_{\nu}<10^{14} \mathrm{eV}$, and $\nu_{x}$ stands for $\nu_{\mu}, \bar{\nu}_{\mu}$ and $\nu_{e}$.

The neutrino spectrum (7) is modified at high energy, where neutrinos are produced by the decay of muons and pions whose life time exceeds the characteristic time for energy loss due to adiabatic expansion and synchrotron emission [33,9]. The synchrotron loss time is determined by the energy density of the magnetic field in the wind rest frame. For the characteristic parameters of a GRB wind, synchrotron losses are the dominant effect, leading to strong suppression of $\nu$ flux above $\sim 10^{16} \mathrm{eV}$. 


\subsubsection{Afterglow neutrinos, $\sim 10^{18} \mathrm{eV}$}

During the transition to self-similarity, protons and electrons are accelerated to high energy in the reverse shocks. High energy protons may interact with the $10 \mathrm{eV}-1 \mathrm{keV}$ photons radiated by the electrons, to produce through pion decay a burst of duration $\sim T$ of ultra-high energy, $10^{17}$ $10^{19} \mathrm{eV}$, neutrinos as indicated by Eq. ([- [10].

While afterglows have been detected in several cases, reverse shock emission has only been identified for GRB 990123 [22]. Both the detections and the non-detections are consistent with shocks occurring with typical model parameters 23, suggesting that reverse shock emission may be common. The predicted neutrino emission depends, however, upon parameters of the surrounding medium that can only be estimated once more observations of the prompt optical afterglow emission are available.

If the density of gas surrounding the fireball is $n \sim 1 \mathrm{~cm}^{-3}$, a value typical to the inter-stellar medium and consistent with GRB 990123 observations, then the synchrotron emission of reverse shock electrons is expected to peak in the $\mathrm{X}$ ray band, $\epsilon_{\gamma}^{b} \approx 1 \mathrm{keV}$, with luminosity $L_{X} \approx$ $2 \times 10^{50} \mathrm{erg} / \mathrm{s} 10$. Using these parameters in Eq. (6), replacing $\Delta t$ with $T \approx 10 \mathrm{~s}$ and recalling that $\Gamma=250$ at the reverse shock stage (see $\S 2$ ), we find $f_{\pi} \approx 0.01$ for protons of energy $10^{19} \mathrm{eV}$. Thus, the expected neutrino fluence for a typical burst, $E \approx 10^{53}$ erg at $z \sim 1$, is [10]

$\epsilon_{\nu}^{2} \Phi_{\nu_{x}} \approx 10^{-4.5}\left(\frac{\epsilon_{\nu}}{10^{17} \mathrm{eV}}\right)^{\alpha} \frac{\mathrm{GeV}}{\mathrm{cm}^{2}}$,

where $\alpha=1 / 2$ for $\epsilon_{\nu}>10^{17} \mathrm{eV}$ and $\alpha=1$ for $\epsilon_{\nu}<10^{17} \mathrm{eV}$. Here too, $\nu_{x}$ stands for $\nu_{\mu}, \bar{\nu}_{\mu}$ and $\nu_{e}$. The value of $\alpha, \alpha=1 / 2$ for $\epsilon_{\nu}>10^{17} \mathrm{eV}$, corresponding to photomeson interactions of photons with energy below the break $\epsilon_{\gamma}^{b} \approx 1 \mathrm{keV}$, differs from the value $\alpha=0$ in the case of internal shocks discussed in §4.1.1, since the low energy, $\epsilon_{\gamma}<\epsilon_{\gamma}^{b}$, photon spectrum is different in the two cases: $d N_{\gamma} / d \epsilon_{\gamma} \propto \epsilon_{\gamma}^{-\beta}$ with $\beta=1$ for internal shocks, and $\beta=3 / 2$ for reverse shocks.

Some GRBs may result from the collapse of a massive star (e.g. [34]), in which case the fireball is expected to expand into a pre-existing wind. For typical wind parameters, the transition to self-similar behavior takes place at a radius where the wind density is $n \approx 10^{4} \mathrm{~cm}^{-3} \gg 1 \mathrm{~cm}^{-3}$. The higher density implies a lower Lorenz factor of the expanding plasma during the transition stage, and hence a larger fraction of proton energy lost to pion production. Protons of energy $\epsilon_{p} \geq 10^{18} \mathrm{eV}$ lose all their energy to pion production in this case, and a typical GRB at $z \sim 1$ is expected to produce a neutrino fluence [10,35]

$\epsilon_{\nu}^{2} \Phi_{\nu_{x}} \approx 10^{-2.5}\left(\frac{\epsilon_{\nu}}{10^{17} \mathrm{eV}}\right)^{\alpha} \frac{\mathrm{GeV}}{\mathrm{cm}^{2}}$,

where $\alpha=0$ for $\epsilon_{\nu}>10^{17} \mathrm{eV}$ and $\alpha=1$ for $\epsilon_{\nu}<10^{17} \mathrm{eV}$.

The neutrino flux is expected to be strongly suppressed at energy $>10^{19} \mathrm{eV}$, since protons are not expected to be accelerated to energy $\gg$ $10^{20} \mathrm{eV}$.

\subsection{Inelastic $p$ - $n$ collisions}

The acceleration, $\Gamma \propto r$, of fireball plasma emitted from the source of radius $r_{0}$ (see $\S 2$ ) is driven by radiation pressure. Fireball protons are accelerated through their coupling to the electrons, which are coupled to fireball photons. Fireball neutrons, which are expected to exist in most progenitor scenarios, are coupled to protons by nuclear scattering as long as the comoving $p$ $n$ scattering time is shorter than the comoving wind expansion time $r / \Gamma c=r_{0} / c$. As the fireball plasma expands and accelerates, the proton density decreases, $n_{p} \propto r^{-2} \Gamma^{-1}$, and neutrons may become decoupled. For $\eta>\eta_{p n}$, where

$\eta_{p n} \approx 400 L_{52}^{1 / 4} r_{0,7}^{-1 / 4}$

and $r_{0}=10^{7} r_{0,7} \mathrm{~cm}$, neutrons decouple from the accelerating plasma prior to saturation, $\Gamma=\eta$, at $\Gamma=\eta_{p n}^{4 / 3} \eta^{-1 / 3}$ [12]. In this case, relativistic relative velocities between protons and neutrons arise, which lead to pion production through inelastic nuclear collisions. Since decoupling occurs at a radius where the collision time is similar to wind expansion time, each $n$ leads on average to one pair of $\nu \bar{\nu}$. The typical comoving neutrino energy, $\sim 50 \mathrm{MeV}$, implies an observed energy $\sim 10 \mathrm{GeV}$. A typical burst, $E=10^{53} \mathrm{erg}$ at $z=1$, with significant neutron to proton ratio and $\eta>400$ will therefore produce a flu- 
ence $F\left(\nu_{e}+\bar{\nu}_{e}\right) \sim 0.5 F\left(\nu_{\mu}+\bar{\nu}_{\mu}\right) \sim 10^{-4} \mathrm{~cm}^{-2}$ of $\sim 10 \mathrm{GeV}$ neutrinos.

Relativistic relative $p$ - $n$ velocities, leading to neutrino production through inelastic collisions, may also result from diffusion of neutrons between regions of the fireball wind with large difference in $\Gamma$ [13]. If, for example, plasma expanding with very high Lorentz factor, $\Gamma>100$, is confined to a narrow jet surrounded by a slower, $\Gamma \sim 10$ wind, internal collisions within the slower wind can heat neutrons to relativistic temperature, leading to significant diffusion of neutrons from the slower wind into the faster jet. Such process may operate for winds with $\eta<400$ as well as for $\eta>400$, and may lead, for certain (reasonable) wind parameter values, to $\sim 10 \mathrm{GeV}$ neutrino flux similar to that due to $p$ - $n$ decoupling in a $\eta>400$ wind.

\subsection{Implications}

The predicted fluence of $\sim 10^{14} \mathrm{eV}$ neutrinos produced by photomeson interactions in internal fireball shocks, Eq. (7), implies a detection probability $\sim 10^{-1.5}$ per burst in planned $1 \mathrm{~km}^{3}$ neutrino telescopes 14, corresponding to detection of several tens of muon induced neutrinos per year correlated in time and direction with GRBs, given the observed GRB rate of $\approx 10^{3} \mathrm{yr}^{-1}$. The predicted fluence of $\sim 10^{17} \mathrm{eV}$ neutrinos, produced by photomeson interactions during the onset of fireball interaction with its surrounding medium, Eqs. (8.91), implies a detection probability $\sim 10^{-4.5}$ in the case of fireball expansion into typical inter-stellar medium gas, and $\sim 10^{-2.5}$ in the case of fireball expansion into a pre-existing massive star wind. Several muon induced neutrinos per year in a $1 \mathrm{~km}^{3}$ detector are expected in the latter case.

Detection of high energy neutrinos will test the shock acceleration mechanism and the suggestion that GRBs are the sources of ultra-high energy protons, since $\geq 10^{14} \mathrm{eV}\left(\geq 10^{18} \mathrm{eV}\right)$ neutrino production requires protons of energy $\geq 10^{16} \mathrm{eV}$ $\left(\geq 10^{19} \mathrm{eV}\right)$. The dependence of $\sim 10^{14} \mathrm{eV}$ neutrino flux on wind Lorentz factor, Eq. (6), and the dependence of $\sim 10^{17} \mathrm{eV}$ neutrino flux on fireball environment, imply that the detection of high energy neutrinos will also provide constraints on wind Lorentz factor [36], and on the GRB progenitor.

Inelastic $p$ - $n$ collisions may produce $\sim 10 \mathrm{GeV}$ neutrinos with a fluence of $\sim 10^{-4} \mathrm{~cm}^{-2}$ per burst, due to either $p$ - $n$ decoupling in a wind with high neutron fraction and high, $>400$, Lorentz factor 11, 12], or to neutron diffusion in a wind with, e.g., strong deviation from spherical symmetry [13]. The predicted number of events in a $1 \mathrm{~km}^{3}$ neutrino telescope is $\sim 10 \mathrm{yr}^{-1}$. Such events may be detectable in a suitably densely spaced detector. Detection of $\sim 10 \mathrm{GeV}$ neutrinos will constrain the fireball neutron fraction, and hence the GRB progenitor.

Detection of neutrinos from GRBs could be used to test the simultaneity of neutrino and photon arrival to an accuracy of $\sim 1 \mathrm{~s}(\sim 1 \mathrm{~ms}$ for short bursts), checking the assumption of special relativity that photons and neutrinos have the same limiting speed. These observations would also test the weak equivalence principle, according to which photons and neutrinos should suffer the same time delay as they pass through a gravitational potential. With $1 \mathrm{~s}$ accuracy, a burst at $100 \mathrm{Mpc}$ would reveal a fractional difference in limiting speed of $10^{-16}$, and a fractional difference in gravitational time delay of order $10^{-6}$ (considering the Galactic potential alone). Previous applications of these ideas to supernova 1987A (see [37] for review), where simultaneity could be checked only to an accuracy of order several hours, yielded much weaker upper limits: of order $10^{-8}$ and $10^{-2}$ for fractional differences in the limiting speed and time delay respectively.

The model discussed above predicts the production of high energy muon and electron neutrinos. However, if the atmospheric neutrino anomaly has the explanation it is usually given, oscillation to $\nu_{\tau}$ 's with mass $\sim 0.1 \mathrm{eV}$ [38], then one should detect equal numbers of $\nu_{\mu}$ 's and $\nu_{\tau}$ 's. Up-going $\tau$ 's, rather than $\mu$ 's, would be a distinctive signature of such oscillations. Since $\nu_{\tau}$ 's are not expected to be produced in the fireball, looking for $\tau$ 's would be an "appearance experiment." To allow flavor change, the difference in squared neutrino masses, $\Delta m^{2}$, should exceed a minimum value proportional to the ratio of source distance and neutrino energy [37]. A burst at 
$100 \mathrm{Mpc}$ producing $10^{14} \mathrm{eV}$ neutrinos can test for $\Delta m^{2} \geq 10^{-16} \mathrm{eV}^{2}, 5$ orders of magnitude more sensitive than solar neutrinos.

\section{REFERENCES}

1. Fishman, G. J. \& Meegan, C. A., ARA\&A 33, 415 (1995).

2. Piran, T., in Unsolved Problems In Astrophysics, eds. J. N. Bahcall and J. P. Ostriker, 343-377 (Princeton, 1996).

3. Kulkarni, S. R. et al., To appear in Proc. of the 5th Huntsville Gamma-Ray Burst Symposium (astro-ph/0002168)

4. Mészáros, P., A\&AS 138, 533 (1999).

5. Waxman, E., Phys. Rev. Lett. 75, 386 (1995).

6. Vietri, M., Ap. J. 453, 883 (1995); Milgrom, M. \& Usov, V., Ap. J. 449, L37 (1995).

7. Waxman, E., Ap. J. 452, L1 (1995).

8. Waxman, E., Physica Scripta T85, 117 (2000); Waxman, E., Nucl. Phys. B (Proc. Suppl.) 87, 345 (2000).

9. Waxman, E., \& Bahcall, J. N., Phys. Rev. Lett. 78, 2292 (1997); Waxman, E., \& Bahcall, J. N., Phys. Rev. D59, 023002 (1999).

10. Waxman, E., \& Bahcall, J. N., Ap. J., in press (hep-ph/9909286).

11. Derishev, E. V., Kocharovsky, V. V., \& Kocharovsky, Vl. V, Ap. J. 521, 640 (1999).

12. Bahcall, J. N., \& Mészáros, P., Phys. Rev. Lett., in press (hep-ph/0004019).

13. Mészáros, P., \& Rees, M., Ap. J., submitted (astro-ph/0007102).

14. Halzen, F., in Proc. 17th International Workshop on Weak Interactions and Neutrinos (Cape Town, South Africa, January 1999) (astro-ph/9904216).

15. Paczyński, B., Ap. J. 308, L43 (1986); Goodman, J., Ap. J. 308, L47 (1986).

16. Paczyński, B. \& Xu, G., Ap. J. 427, 708 (1994); Mészáros, P., \& Rees, M., MNRAS 269, 41P (1994).

17. Woods, E. \& Loeb, A., Ap. J. 453, 583 (1995).

18. Bhat, P. N., et al., Nature 359, 217 (1992).

19. Krolik, J. H. \& Pier, E. A., Ap. J. 373, 277 (1991); Baring, M., Ap. J. 418, 391 (1993).

20. Blandford, R. D., \& Mckee, C. F., Phys. Flu- ids 19, 1130 (1976).

21. Blandford, R., \& Eichler, D., Phys. Rep. 154, 1 (1987).

22. Akerlof, C. W. et al., Nature, 398, 400 (1999).

23. Sari, R. \& Piran, T., Ap. J. 517, $L 109$ (1999); Mészáros, P. \& Rees, M., MNRAS 306, L39 (1999).

24. Krumholtz, M., Thorsett, S. E., \& Harrison, F. A., Ap. J. 506, L81 (1998); Hogg, D. W. \& Fruchter, A. S., Ap. J. 520, 54 (1999).

25. Freedman, D. L., \& Waxman, E., submitted to Ap. J. (astro-ph/9912214).

26. Mao, S. \& Mo, H. J., A\&A 339, L1 (1998); Porciani, C., \& Madau, P., submitted to Ap. J. (astro-ph/0008294).

27. Bird, D. J., et al., Ap. J. 424, 491 (1994).

28. Efimov, N. N. et al., in Proceedings of the International Symposium on Astrophysical Aspects of the Most Energetic Cosmic-Rays, edited by M. Nagano and F. Takahara (World Scientific, Singapore, 1991), p. 20.

29. Takeda, M. et al., Phys. Rev. Lett. 81, 1163 (1998).

30. Gaisser, T. K. et al., Phys. Rev. D47, 1919 (1993); Dawson, B. R., Meyhandan, R., Simpson, K.M., Astropart. Phys. 9, 331 (1998).

31. Greisen, K., Phys. Rev. Lett. 16, 748 (1966); Zatsepin, G. T., \& Kuzmin, V. A., JETP Lett., 4, 78 (1966).

32. Waxman, E., \& Bahcall, J. N., Ap. J. in press (hep-ph/9912326)

33. Rachen, J. P., \& Mészáros, P., Phys. Rev. D58, 123005 (1998).

34. Woosley, S. E., \& MacFadyen, A. I., A\&AS, 138, 499 (1999); Chevalier, R. A., \& Li, Z., Ap. J., 536, 195 (2000).

35. Dai, Z. G., \& Lu, T., submitted to Ap. J. astro-ph/0002430.

36. Alvarez-Muniz, J., Halzen, F., \& Hooper, D. W., Phys. Rev. D, in press astro$\mathrm{ph} / 0006027)$.

37. Bahcall, J. N., Neutrino Astrophysics, Cambridge University Press (NY 1989).

38. Fukuda, Y., et al., Phys. Lett. B335, 237 (1994); Casper, D., et al., Phys. Rev. Lett. 66, 2561 (1991); Fogli, G. L., \& Lisi, E., Phys. Rev. D52, 2775 (1995). 